Chang-Seok Ki • Sun-Young Kong • Dae Won Seo

Seung Bong Hong • Hyung-Jin Kim • Jong-Won Kim

\title{
Two novel mutations in the EPM2A gene in a Korean patient with Lafora's progressive myoclonus epilepsy
}

\begin{abstract}
The progressive myoclonus epilepsy of the Lafora type (LD; MIM 254780) is a rare autosomal recessive disorder characterized by epilepsy, myoclonus, progressive neurological deterioration, and the presence of periodic acid-Schiff-positive polyglucosan inclusions (Lafora bodies). Mutations in the EPM2A gene have recently been found to cause LD and about 30 or more mutations have been reported thus far. LD is relatively common in countries of the Mediterranean Basin, the Middle East, India, and Pakistan. Although a few sporadic cases with the typical LD phenotype have also been reported in the Far East including Korea and Japan, a recent effort to find mutations in Japanese LD families was not successful. In the present study, we report two novel mutations in a Korean girl with LD; a 1-bp insertion mutation (c.223insC; G75fsX107) in exon 1 and a missense mutation (c.559A $>\mathrm{G}$; T187A) in exon 3 of the EPM2A gene. To our knowledge, this is the first report of a genetically confirmed case of LD in Koreans and also in the Far East.
\end{abstract}

Key words Progressive myoclonus epilepsy $(\mathrm{PME}) \cdot$ Lafora disease - Lafora body - EPM2A - Mutation analysis . Insertion mutation $\cdot$ Missense mutation $\cdot$ Korean

\section{Introduction}

Lafora disease (LD; MIM 254780) is a rare autosomal recessive disorder characterized by epilepsy, myoclonus, pro-

C.-S. Ki · S.-Y. Kong $\cdot$ J.-W. Kim $(\bowtie)$

Department of Clinical Pathology, Sungkyunkwan University

School of Medicine, Samsung Medical Center, 50 Ilwon-Dong,

Kangnam-Gu, Seoul 135-710, Korea

Tel. +82-2-3410-2705; Fax +82-2-3410-2719

e-mail: culture@med.skku.ac.kr

D.W. Seo $\cdot$ S.B. Hong $\cdot$ H.-J. Kim

Department of Neurology, Sungkyunkwan University School of

Medicine, Samsung Medical Center, Seoul, Korea

The third and sixth authors contributed equally to this work. gressive neurological deterioration, and the presence of glycogen-like intracellular inclusion bodies (Lafora bodies) (Minassian 2001). Recently, mutations in the EPM2A gene at 6q24 encoding a dual-specificity phosphatase (laforin) have been found to be responsible for up to $80 \%$ of LD cases and about 30 or more mutations have been reported thus far (Ganesh et al. 2002; Gomez-Garre et al. 2000; Minassian et al. 1998, 2000a, 2000b; Serratosa et al. 1999). LD occurs worldwide but is relatively common in the Mediterranean countries of southern Europe, the Middle East, India, Pakistan, and northern Africa (Delgado-Escueta et al. 2001). Although a few sporadic cases with the typical LD phenotype have also been reported in the Far Eastern countries, including Korea, Japan, and China, there has been no report of a genetically confirmed case. Recently, Ganesh et al. (2001) screened for mutations in the EPM2A gene in four Japanese LD families but failed to identify any mutations that cosegregated with the LD phenotype. They suggested that EPM2A was not involved in the disease phenotype of the families studied and that locus heterogeneity for LD might exist in the Japanese population. Recently, we identified a Korean girl with typical clinical features of LD and performed a mutation analysis to test whether the patient had EMP2A gene mutations. We herein describe the clinical features of the patient and present two novel mutations of the EPM2A gene.

\section{Patients and methods}

Case report

A 15-year-old Korean girl presented with intractable seizures since the age of 10 years. Her seizures started with visual or auditory auras, which were sometimes followed by generalized tonic-clonic seizures. In addition, she had generalized myolonic seizures since the age of 13 years. Despite antiepileptic treatment with valproate $(1800 \mathrm{mg} /$ day), clonazepam ( $3 \mathrm{mg} /$ day), lamotrigine ( $400 \mathrm{mg} /$ day), and zonisamide $(400 \mathrm{mg} / \mathrm{day})$, the seizures were poorly con- 


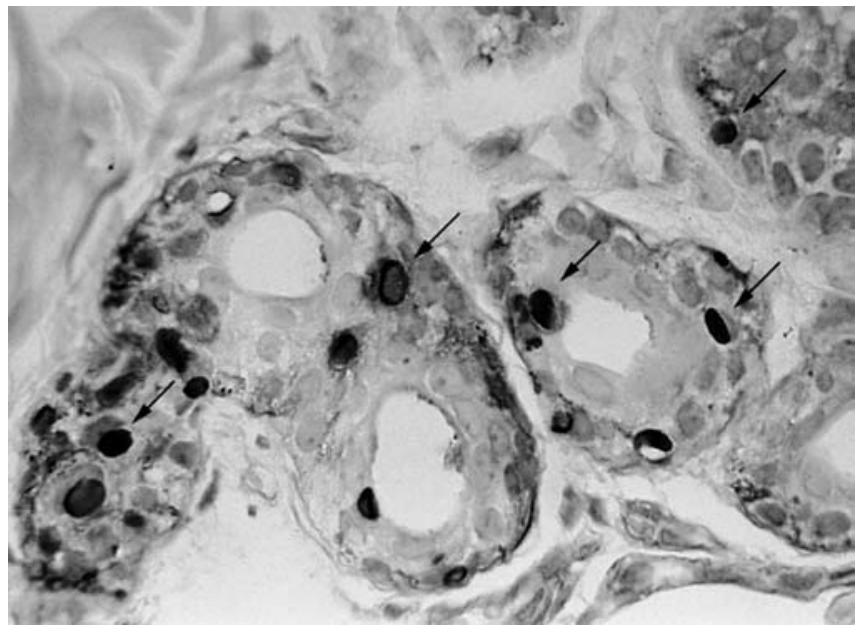

Fig. 1. Numerous periodic acid-Shiff (PAS)-positive round or oval inclusions are present in the epithelia of sweat gland duct cells (arrows; axillary skin biopsy, PAS stain, $\times 400$ )

trolled. She had experienced a simple febrile seizure at the age of 3 years, but there was no history of a perinatal insult, encephalitis, metabolic diseases, or head trauma. She was born in Seoul, Korea and showed normal development during childhood years. There was no family history of a seizure and her parents were not consanguineous. On neurological examination, she had no definite abnormalities of cognitive function, motor ability, sensation, reflexes, or gait. Laboratory tests including complete blood count, blood chemistry, and urinanalysis were not remarkable. Additional studies to rule out metabolic disorders including Gaucher disease were normal. Electroencephalography showed a slowing of background activity and very frequent discharges of spikes, polyspikes, and generalized spike waves. Numerous periodic acid-Schiff-positive Lafora bodies were observed in the cytoplasms of sweat gland duct cells in the axillary skin biopsy (Fig. 1).

\section{Molecular genetic study}

After we obtained informed consent, blood samples were collected from the patient and her mother. Because the patient's father refused the mutation analysis, we could not obtain his sample. Genomic DNA was isolated from peripheral blood leukocytes using a Wizard genomic DNA purification kit according to the manufacturer's instruction (Promega, Madison, WI, USA). All four exons of the EPM2A gene were amplified by polymerase chain reaction (PCR) using primers designed by Serratosa et al. (1999) or by the authors. Cycle sequencing was performed with a BigDye Terminator Cycle Sequencing Ready Reaction kit (Applied Biosystems, Foster City, CA, USA) on the ABI 377 or the ABI 3100 Genetic Analyzer (Applied Biosystems). The mutations found in the EPM2A gene were further confirmed by the PCR-restriction fragment length polymorphism (RFLP) method.

\section{Results}

Two novel mutations of the EPM2A gene were identified. One was a heterozygous 1-bp insertion mutation (c.223insC; G75fsX107) in exon 1 and the other was a heterozygous missense mutation (c.559A $>\mathrm{G}$; T187A) in exon 3 of the EPM2A gene (Fig. 2). The patient's mother was a heterozygous carrier for the T187A mutation and did not have the c.223insC mutation. We could not determine whether the c.223insC mutation was de novo or inherited because the patient's father was not tested.

The c.223insC mutation introducing a novel AvaII site in the amplified DNA was confirmed by the PCR-RFLP method and was not found in 100 unrelated control subjects (Fig. 2). Because the T187A mutation neither created nor destroyed a restriction site, we designed a mismatch primer ( $5^{\prime}$-actgaagcatgaattggggaCt-3'; underlined sequence indicates mismatched site) to introduce a PstI site in the amplified DNA. The reverse primer was 5'tacagcaaggaggcagaaca- $3^{\prime}$. With this method, the T187A mutation was confirmed in the patient and her mother, and was not found in 100 unrelated control subjects (Fig. 2).

\section{Discussion}

LD is relatively common in countries of the Mediterranean Basin, the Middle East, Malaysia, Indonesia, India, and Pakistan (Delgado-Escueta et al. 2001). In Far Eastern countries, including Korea, China, and Japan, LD is extremely rare in that only a few cases with the typical clinical features of LD have been reported in the Japanese population, but not in the Chinese population (Ganesh et al. 2001). In Koreans, only two brothers with LD in one family have been reported, who were diagnosed by the typical clinical features and by the presence of Lafora bodies (Lee et al. 1995). However, there has been no report of a genetically confirmed LD in the Far East and a recent effort to identify mutations in the EPM2A gene in four Japanese LD families was not successful (Ganesh et al. 2001).

It is not clear why the Japanese LD patients do not have any mutations in the EPM2A gene, however, locus heterogeneity might be an explanation. Minnassian et al. (1999) examined for heterogeneity through the admixture test in 22 families and estimated the proportion of linked families to be $75 \%$ to $85 \%$. Therefore, mutations in at least one still unknown gene also cause LD, and this gene might be responsible for the Japanese LD families. Alternatively, defects in a still uncharacterized regulatory region of the EPM2A gene or in critical enhancer elements thereof might produce the LD phenotype. This possibility was raised because five LD families with 6q24 haplotypes and homozygosities did not have any mutations in the coding region of the EPM2A gene (Ganesh et al. 2002).

The EPM $2 A$ gene encodes a dual-specificity phosphatase, laforin, and is composed of four exons. The laforin contains an N-terminal carbohydrate-binding domain 
Fig. 2A-D. Identification of EPM $2 A$ gene mutations. A Direct sequencing analysis demonstrated a heterozygous 1-bp insertion (arrow; c.223insC) resulting in a G75fsX107 mutation in exon 1. Because the sequencing was performed with an antisense primer, overlapped peaks appear from the $\mathrm{T}+\mathrm{G}$ peaks (arrow). B Polymerase chain reaction-restriction fragment length polymorphism (PCR-RFLP) analysis confirmed the c.223insC mutation, which introduced an AvaII site, creating 209- (arrow) and 98-bp (hardly visible) bands. C A heterozygous A-to-G transition (arrow; c.559A $>\mathrm{G}$ ) resulting in a T187A missense mutation is observed in exon 3. D A mismatch PCR-RFLP analysis also confirmed the T187A mutation, which introduced a PstI site, creating 191(arrow) and 25-bp (not visible) bands. Con, Wild-type control; Pat, patient; $M$, 100-bp size marker
(A)

(B)
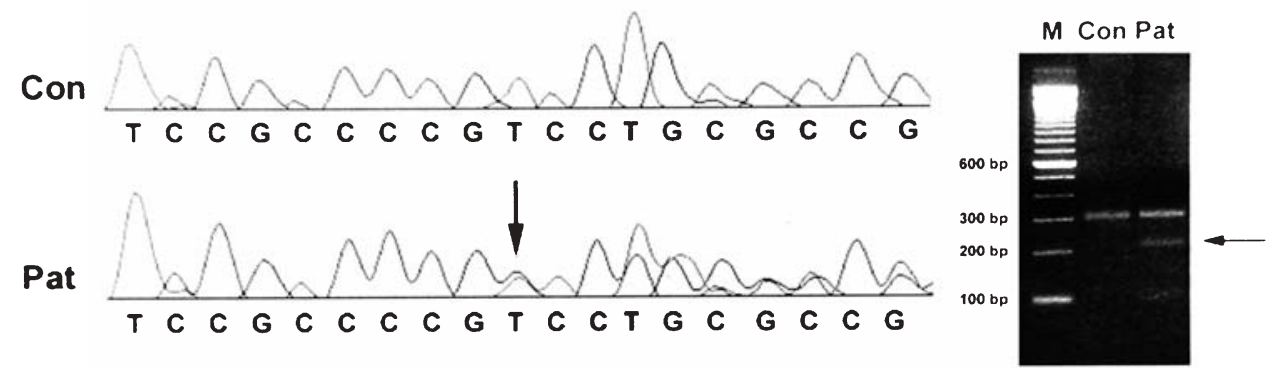

(C)

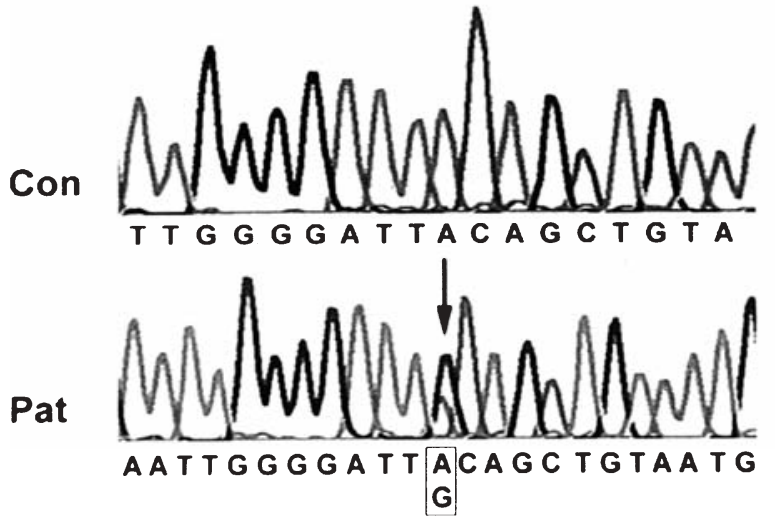

(D)

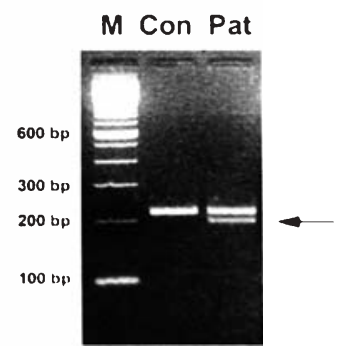

(CBD-4) coded mainly by exon 1 and the dual-specificity phosphatase domain (DSPD) spanning exons 3 and 4 (Ganesh et al. 2000). Recently, Ganesh et al. (2002) proposed that two subsyndromes of LD might exist: (1) classical LD with adolescent-onset stimulus-sensitive grand mal, absence, and myoclonic seizures followed by dementia and neurological deterioration, and associated with mutations in exon 4; and (2) atypical LD with childhood-onset dyslexia and learning disorder followed by epilepsy and neurological deterioration, and associated mainly with mutations in exon 1. Ganesh et al. (2002) also provided clues for distinct roles for the CBD-4 and DSPD of laforin in the etiology of the two subsyndromes of LD, demonstrating that mutations affecting the functions of DSPD, and not of CBD-4, led to classical LD and those affecting both domains developed atypical LD.

The patient described in the present study was a compound heterozygote carrying the c.223insC (G75fsX107) mutation in exon 1 and the c.559A $>\mathrm{G}$ (T187A) mutation in exon 3 of the EPM2A gene, and could be classified as having a classical LD because she did not show childhoodonset dyslexia and learning disorder. It is not clear why she showed classical LD despite the c.223insC mutation affecting the functions of CBD-4. Because there are few data describing a compound heterozygous LD patient affecting both CBD-4 and DSPD, and because not all patients with mutations affecting CBD-4 showed classical LD (Ganesh et al. 2002), more data are mandatory to understand the genetic background of the two subsyndromes.

Because we could not test the patient's father, it was difficult to exclude the possibility that two mutations found in the patient might be located in one chromosome. How- ever, because the patient's mother was a heterozygous carrier of the T187A mutation and did not have the c.223insC mutation, de novo introduction of the c.223insC mutation to the same chromosome already carrying the T187A mutation is unexpected. In summary, we identified two novel mutations in the EPM2A gene in a Korean girl with the typical phenotype of LD, which we believe would improve our understanding of the genotype-phenotype correlations of EPM2A mutations. To our knowledge, this is the first report of a genetically confirmed case of LD in Koreans and also in the Far East.

Acknowledgments This work was supported by the fund from the Center for Functional Analysis of Human Genome (FG-2-1-02), Korea.

\section{References}

Delgado-Escueta AV, Ganesh S, Yamakawa K (2001) Advances in the genetics of progressive myoclonus epilepsy. Am J Med Genet 106:129-138

Ganesh S, Agarwala KL, Ueda K, Akagi T, Shoda K, Usui T, Hashikawa T, Osada H, Delgado-Escueta AV, Yamakawa K (2000) Laforin, defective in the progressive myoclonus epilepsy of Lafora type, is a dual-specificity phosphatase associated with polyribosomes. Hum Mol Genet 9:2251-2261

Ganesh S, Shoda K, Amano K, Uchiyama A, Kumada S, Moriyama N, Hirose S, Yamakawa K (2001) Mutation screening for Japanese Lafora's disease patients: identification of novel sequence variants in the coding and upstream regulatory regions of EPM2A gene. Mol Cell Probes 15:281-289

Ganesh S, Delgado-Escueta AV, Suzuki T, Francheschetti S, Riggio C, Avanzini G, Rabinowicz A, Bohlega S, Bailey J, Alonso ME, Rasmussen A, Thomson AE, Ochoa A, Prado AJ, Medina MT, 
Yamakawa K (2002) Genotype-phenotype correlations for EPM2A mutations in Lafora's progressive myoclonus epilepsy: exon 1 mutations associate with an early-onset cognitive deficit subphenotype. Hum Mol Genet 11:1263-1271

Gomez-Garre P, Sanz Y, Rodriguez De Cordoba SR, Serratosa JM (2000) Mutational spectrum of the EPM2A gene in progressive myoclonus epilepsy of Lafora: high degree of allelic heterogeneity and prevalence of deletions. Eur J Hum Genet 8:946-954

Lee SH, Lee H, Yi SD, Lim JG, Lee DK, Park YC (1995) Two cases of Lafora's disease. Korean J Neurol 13:96-103

Minassian BA (2001) Lafora's disease: towards a clinical, pathologic, and molecular synthesis. Pediatr Neurol 25:21-29

Minassian BA, Lee JR, Herbrick JA, Huizenga J, Soder S, Mungall AJ, Dunham I, Gardner R, Fong CY, Carpenter S, Jardim L, Satishchandra P, Andermann E, Snead OC 3rd, Lopes-Cendes I, Tsui LC, Delgado-Escueta AV, Rouleau GA, Scherer SW (1998) Mutations in a gene encoding a novel protein tyrosine phosphatase cause progressive myoclonus epilepsy. Nat Genet 20:171-174
Minassian BA, Sainz J, Serratosa JM, Gee M, Sakamoto LM, Bohlega S, Geoffroy G, Barr C, Scherer SW, Tomiyasu U, Carpenter S, Wigg K, Sanghvi AV, Delgado-Escueta AV (1999) Genetic locus heterogeneity in Lafora's progressive myoclonus epilepsy. Ann Neurol 5:262-265

Minassian BA, Ianzano L, Delgado-Escueta AV, Scherer SW (2000a) Identification of new and common mutations in the EPM2A gene in Lafora disease. Neurology 54:488-490

Minassian BA, Ianzano L, Meloche M, Andermann E, Rouleau GA, Delgado-Escueta AV, Scherer SW (2000b) Mutation spectrum and predicted function of laforin in Lafora's progressive myoclonus epilepsy. Neurology 55:341-346

Serratosa JM, Gomez-Garre P, Gallardo ME, Anta B, de Bernabe DB, Lindhout D, Augustijn PB, Tassinari CA, Malafosse RM, Topcu M, Grid D, Dravet C, Berkovic SF, de Cordoba SR (1999) A novel protein tyrosine phosphatase gene is mutated in progressive myoclonus epilepsy of the Lafora type (EPM2). Hum Mol Genet 8:345352 